\title{
Image De-noising By Decision Based Expanded Window Median Filter Using Multiple Scanning
}

\author{
Reva Sethi ${ }^{1}$, Vishal Kumar Arora ${ }^{2}$ \\ ${ }^{1}$ (Department of CSE, SBS State Technical Campus, Ferozepur, India) \\ ${ }^{2}$ (Department of CSE, SBS State Technical Campus, Ferozepur, India)
}

\begin{abstract}
This paper proposes a new filter for noisy imagescorrupted with salt and pepper noise which are caused due to flaws in sensor, transmission. Proposed algorithm (Decision Based Expanded Window Median Filters (DBEWMF) with multiple scanning) works on noisy pixel and noise free pixel left unchanged. Filteruses an expanded window, where processed pixel is a central pixel follows with multiple scanning of same image. Filter Expands the window size (up to $7 \times 7$ ), if window contains noisy pixels equal to or more than three forth of total pixels in orderto find more noise free pixels if still window has more noisy pixels than it will place as it is in de-noise image in first and second scanning but in third scanning replacement of processed pixel with mean value of window else window contains less than three forth noisy pixels than processed pixel is replaced using median value of window. The Proposed scheme shows better quantitatively and qualitatively in the image than standard and other algorithm.
\end{abstract}

Keywords: Decision based noise removal (Three levels), Expandedwindow, Median filters, Salt and pepper noise.

\section{Introduction}

Images are often corrupted by Salt and Pepper noise which is caused due to bit error during transmission of the image signal or introduce during image acquisition stage. Salt and pepper noise has only two intensity values such as 0 and 255 's. Thus, this noise may corrupt Image quality or some time loss of fine details of image[1].This noise randomly changes intensities of some other pixels to the maximum and minimum values of the intensity range on the image. Many nonlinear filters[2] have been proposed for restoration of the images corrupted by Salt and Pepper noise. Widely used nonlinear digital filter is Median filter because of its capability of removing Salt and Pepper noise and other noises by preserving image boundaries. For a noisy image $\mathrm{I}(\mathrm{i}, \mathrm{j})$ degraded by salt and pepper noise Median filtering operation can be mathematically written as.

$K(i, j)=\operatorname{median}\{I(i, j),(i, j) € W\}$

Where $K(I, j)$ is a restored image and $\mathrm{W}$ represents a spatial window around a pixel, on any location $(\mathrm{i}, \mathrm{j})$.

\section{Related Work}

Standard Median Filter (SMF) was a good method to remove Salt and Pepper noise but cause blurring at large window size.it has been noticed that this method works good only at low noise density and computaional efficiency[3,4]

Median filters operates only on noisy pixels and niosefree pixels kept unchanged so it is needed to indentify[5] a pixel whether it is noisy or noise free before filtering.To overcome the drawback of SMF filter various Adaptive mean Filters(AMF)[6,7], Decision Based Algorithm(DBA) have been proposed. In these filters firstly noisy pixels are detected and then replaced with median value without any change in noise free pixels.AMF Perform effective at low noise density but in case of high noise density[10,11], smallwindow size may brings blurring of image details. This filters also not deal with the local features of the image.To overcome the problem of this algorithm Decision Based Algorithm (DBA) has been proposed[1]. DBA starts filteration when noisy pixel is identified means either 0 or 255 . It uses a fixed window size of dimension $3 \times 3$.Replacement of noisy pixels iswith the median value of window on the base of predefined condition. Algorithm posses a serious problem at high noise density because the median value is 0 or 255 which is also anoisy pixel. In such situations this algorithm uses neighboring pixelsfor replacement. This repeated replacement of neighboring pixels leads to streaking effect. This problem is overcome by Modified Decision based unsymmetrical trimming filters(MDBUTMF) $[12,9]$. This algorithmconsiders a fixed window of size $3 \times 3$ for denoising purpose. At low noise density unsymmetrical trimming is used and then replace the noisy pixel withthe median but at high noise density replacement with mean directly.However,at high noise density the probability of the situation that the entire pixels are noisy is high. This replacement produces dark patch[14]like surface in restored image.

In Decision Based Coupled Window Median Filter(DBCWMF) problem of patches has been overcome using coupled window of increasing dimension[8], is to increase the probability of finding noise free pixel. In this algorithm pixel is indentified(noisy or noise free) if the pixel is noisy then filtering is performed by 
selecting window 3x3. Unsymmetrical Trimming Median Filters are also used thattrims all 0's and 255's pixel from the window and then calculate medain value which is replaced with the processing pixel.This approch is also not perform well to high noise density so window of increasing dimension is used. Selected window is checked for a condition either all pixels are noisy or not.if condition is false unsymmetrical trimming median filters is applied that replace the central noisy pixel with the median value of pixels which are left after trimming. If condition is true it increases wndow size till $7 \times 7$ and after this replace the cental noisy pixel with the mean of the pixels using mean filters[16] which may generate dark patches because probability of finding noisy pixels is moreat high noise density.

\section{Proposed Algorithm}

In proposed algorithm Decision Based Expanding Window Median Filter(DBEWMF) With Multiple scanning prefers median filtering rather than mean filtersat high noise density because median filters are better compares to mean filters because with multiple scanning probability of find noisy pixel get reduced at high noise density. This algorithm prefers some other conditions then DBCWMF and gives better result.In proposed algorithm in

First Scanning first step is to detect the impulsive noiseThe processing pixel is checked whether it is noisy or noise free that is if the processing pixel lies between 0 and 255 than it is noise free pixel, it is left unchanged. If the processing pixel is 0 or 255 than it is noisy pixel filtering is performed by selecting window around the processed pixel. Now,window is checked for a condition that is it contains noisy pixel which are more than or equal to three forth( $3 / 4)$ of total pixel. If condition is true than window size will be increased up to $7 \times 7$ to find noise free pixels but if still noisy pixels are more then algorithm will palce the noisy pixel as it is in the denoisy image without any replacement. On the other hand if the condition becomes false Unsymmetrical trimmimg Filters trims all 0 's and 255's from the window and repalce the noisy(central)pixel of the window with the median value of window. First scanning genetares a restored image as shown in figure 1. This denoised image is taken as input in Second Scanning and perform all actions which are performed in first scanning. Second Scanning will also generate a restored image used in third scanning. In third scanning when noisy pixel is found processing will be applied. All step are same till increasing the window size upto $7 \mathrm{x} 7$ but if still condition is true then filter will replace the central noisy pixel with the mean of the selected window.A denoisy image will be generated(as shown in figure 2) by the propsed algorithm which have better quality and other metrics.

\section{At First stage}

Step 1: Select a 2-D window $W_{n}$ of size $(2 n+1) \times(2 n+1)$ and assume that pixel being processed is $I(i, j)$ of window $\mathrm{W}_{\mathrm{n}}$ expanded window(let $\left.\mathrm{n}==1\right)$

Step 2: If $0<I(i, j)<255$ then $I(i, j)$ is a noise free pixel so it should be left as it is. It can be written as:

$K(i, j)=\left\{\begin{array}{c}\text { noise free pixel if } 0<I(i, j)<255 \\ \text { noisy pixel if } I(i, j)=0 \text { or } 255\end{array}\right.$

If $I(i, j)=0$ or 255 then it is noisy pixel and should be processed.

Step 3: If a pixel is noisy then DBEWMF with multiple scanning filter will use window of neighboring pixels (selected in Step 1)

Case 1: If $W_{n}$ does not contain noisy pixels equal to or more than $3 / 4$ of total no of pixels then unsymmetrically trim all 0 's an 255 's from the window $\mathrm{W}_{\mathrm{n}}$, a new trimmed window $\mathrm{TW}_{\mathrm{n}}$ is there. $\mathrm{IfTW}_{\mathrm{n}}$ contains non-zero elements, then $I(I, j)$ can be replaced as:

$\mathrm{K}(\mathrm{i}, \mathrm{j})=\left\{\operatorname{median}\left(\mathrm{TW}_{\mathrm{n}}\right)\right\}$

where $K(\mathrm{i}, \mathrm{j})$ is a restored value

Case 2: If $\mathrm{W}_{\mathrm{n}}$ contains noisy pixels equal to or more than $3 / 4$ of total no of pixels then update the value of $\mathrm{n}$ as below $(\mathrm{n}<5)$ :

$\mathrm{n}=\mathrm{n}+1$ and go to step

Step 4: $W_{n}$ can be increase up to $n<5$ for finding the noise free pixels because beyond $n \leq 5$ will increase the computational complexity of algorithm. At $n=4$ filter will place as its in to the restored value without any replacement as:

$\mathrm{K}(\mathrm{i}, \mathrm{j})=\mathrm{I}(\mathrm{i}, \mathrm{j})$ 
Step 5: Repeat step 1-4 in for loop until all the pixels of the image are processed.

First stage give a restored image as output. This restored image will be the input for Second stage.

\section{At First Two Stages}

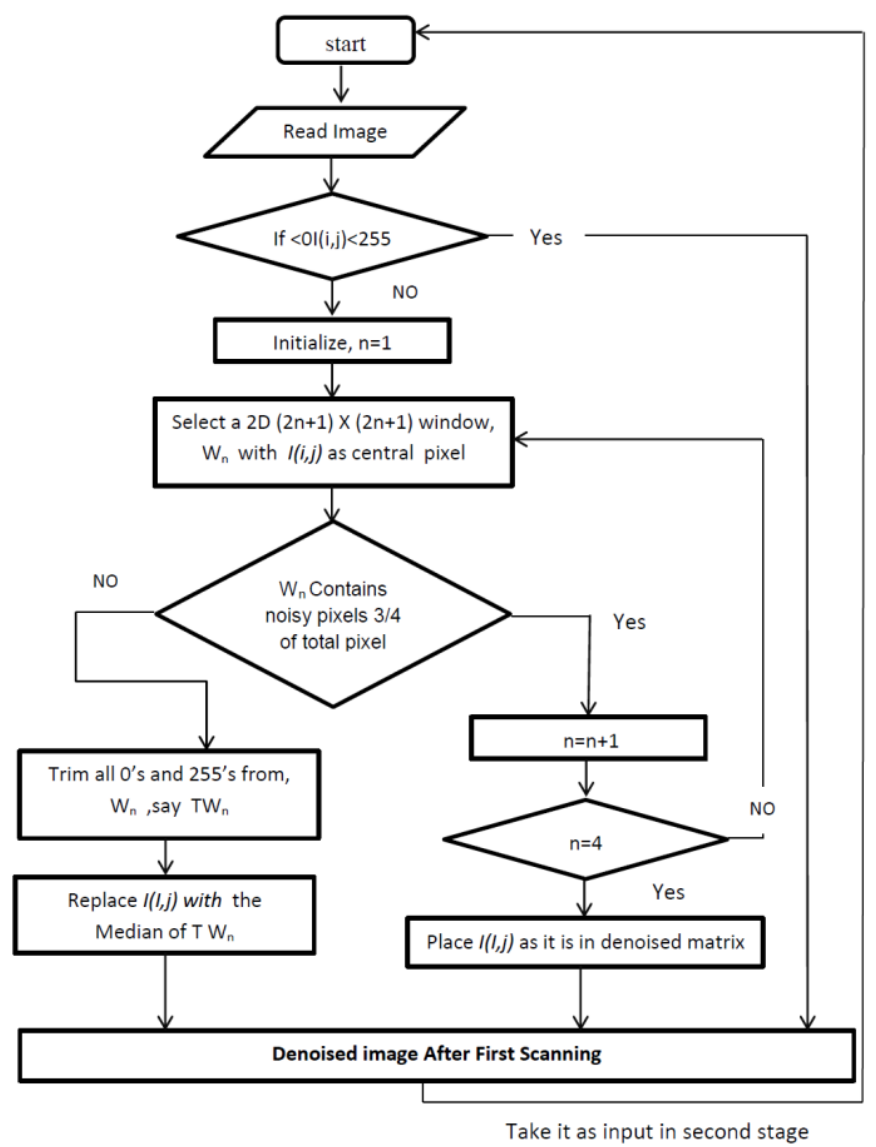

Figure 1: Flowchart of DBEWMF At First\& Second Stage

At Second Stage

Take restored image after first scanning as input. Perform all steps as it in first stage. Restored image at this stage is input for $3^{\text {rd }}$ stage. Flow chart is also same as in First stage

At Third Stage

Take restored image after second scanning as input. Repeat all Steps from 1to3 steps as in First stage.

\section{Step 4:}

$\mathrm{W}_{\mathrm{n}}$ can be increase up to $\mathrm{n}<5$ for finding the noise free pixels. At $\mathrm{n}=4$ filter will replace noisy pixel with mean of window.

$\mathrm{K}(\mathrm{i}, \mathrm{j})=\left\{\right.$ mean $\left.\left(\mathrm{W}_{1}\right)\right\}$

Step 5: Repeat step 1-4 in for loop until all the pixels of the image are processed.

For a RGB image the above mentioned algorithm need to separately operate on each color channel. After denoising operation separate channels can be concatenate to have a denoised color image. 


\section{At Third Stage}

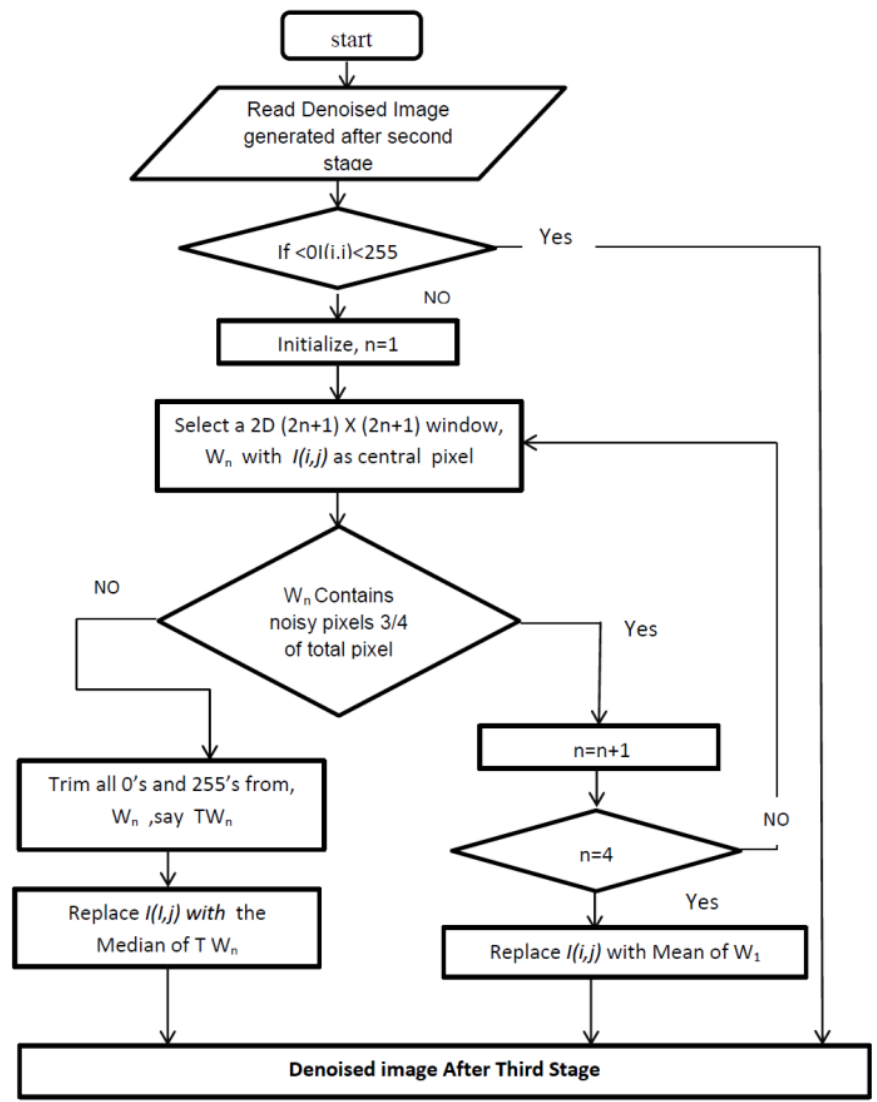

Figure 2: Flowchart of DBEWMF at Third Stage

\section{Results and Discussion}

Denoising performance of DBEWMF with multiple scanning has been evaluated on the basis of quantitative performance criteria of Mean square error (MSE), Bit error rate (BER), Peak signal-to-noise ratio (PSNR), Image enhancement factor (IEF), Structural similarity index measure (SSIM), Image Quality Index(IQI). Equations are given below:

1) The MSE (mean square error):Defined it asaverage squared difference between an original image and a restored image. It is calculated as[15]:

$$
\text { MSE }=\frac{1}{A B} \sum_{i=1}^{A} \sum_{j=1}^{B}(i(i, j)-k(i, j))^{2}
$$

2) The BER (bit error ratio):Defined it as the ratio that describes how many bits received in error over the number of the total bits received. It is often expressed as percentage and calculated by comparing bit values of restored image and original image.

$\mathrm{BER}=\mathrm{P} /(\mathrm{A} * \mathrm{~B})$

3) The PSNR (peak signal to noise ratio):It is a quality metric used to determine the degradation in restored image with respect to the original image or also defined as ratio between maximum power of a signal and power of distorted signal. It is most easily defined via the mean squared error (MSE) as[13]:

$\mathrm{PSNR}=10 \log _{10} \frac{\mathrm{Q} * \mathrm{Q}}{\mathrm{MSE}}$

4) The SSIM (structural similaraity index ): SSIM is used to measure the similarity between two images.SSIM is designed to improve on traditional methods like peak signal to noise ratio (PSNR) and mean squared error (MSE), which have proven to be inconsistent with human eye perception. It is calculated by formula given below[15] 
$\operatorname{SSIM}(I, K)=\frac{\left(2 \mu_{\mu} \mu_{K}+c 1\right)\left(2 \sigma_{I K}+c 2\right)}{\left(\mu_{I}^{2}+\mu_{\mathrm{K}}^{2}+c 1\right)\left(\sigma_{I}^{2}+\sigma_{\mathrm{K}}^{2}+c 2\right)}$

Where I original image, $\mathrm{K}$ restored image with $\mu_{\mathrm{I}}$ the average ofl,

$\mu_{\mathrm{K}}$ the average of $\mathrm{K}$

$\mathrm{C}_{1}$ and $\mathrm{C}_{2}$ being the constants

and $\sigma_{\mathrm{I}}^{2}$ the variance of $\mathrm{I}, \sigma_{\mathrm{K}}^{2}$ the variance of $\mathrm{K}$,

5) IQI (Image Quality Index): Algorithm performance has also been evaluate on qualitative basis as image quality index (IQI) and visual perceptionIQI is calculated by modeling any image distortion as a combination of three factors: loss of correlation, luminance distortion, and contrast distortion. IQI is calculated using:

$\mathrm{IQI}=\operatorname{Corr}(\mathrm{I}, \mathrm{K}) \times \operatorname{Lum}(\mathrm{I}, \mathrm{K}) \times \operatorname{Cont}(\mathrm{I}, \mathrm{K})$

Corr $(\mathrm{I}, \mathrm{K})=\frac{\sigma_{\mathrm{IK}}}{\sigma_{\mathrm{I} \sigma_{\mathrm{K}}}}$

$\operatorname{Lum}(\mathrm{I}, \mathrm{K})=\frac{2 \mu_{\mathrm{I}} \mu_{\mathrm{K}}}{\mu_{1}^{2}+\mu_{\mathrm{K}}^{2}}$

$\operatorname{Cont}(\mathrm{I}, \mathrm{K})=\frac{2 \sigma_{\mathrm{I}} \sigma_{\mathrm{K}}}{\sigma_{\mathrm{I}}^{2}+\sigma_{\mathrm{K}}^{2}}$

The allowed range of IQI is $[-1,1]$. Its 1 value means restored image is equal to original image and consider as best value.

6) IEF(Image Enhancement Factor): IEF is related to enhancement of restored image after filtering. IEF depends on original image, noisy image and restored image and is calculated as:

$\operatorname{IEF}=\frac{\sum_{\mathrm{i=1}}^{\mathrm{A}} \sum_{\mathrm{j}=1}^{\mathrm{B}}[\mathrm{X}(\mathrm{i}, \mathrm{j})-\mathrm{I}(\mathrm{i}, \mathrm{j})]^{2}}{\sum_{\mathrm{i}=1}^{\mathrm{A}} \Sigma_{\mathrm{j}=1}^{\mathrm{B}}\left[\mathrm{K}(\mathrm{i}, \mathrm{j})-\left.\mathrm{I}(\mathrm{i}, \mathrm{j})\right|^{2}\right.}$

where $I(i, j), X(i, j)$, and $K(i, j)$ represent original, noisy and restored image of dimension $A \times B$ and $P$ is the count number whose initial value is zero and it increments by one if there is any bit difference between Original and restored image. Q denotes the peak signal value of the cover image which is equal to 255 for 8 bit images.

\section{Figures and Tables}

We have used Matlab R2010 as simulation tool. In present study standard image Lena(512 X 512)has been used. The varying Noise density is ranging from $10 \%$ to $90 \%$.Better performance of proposed algorithm DBEWMF with multiple scanning over DBCWMF has been proven through the simulation results and performance graph only for Lena image as below

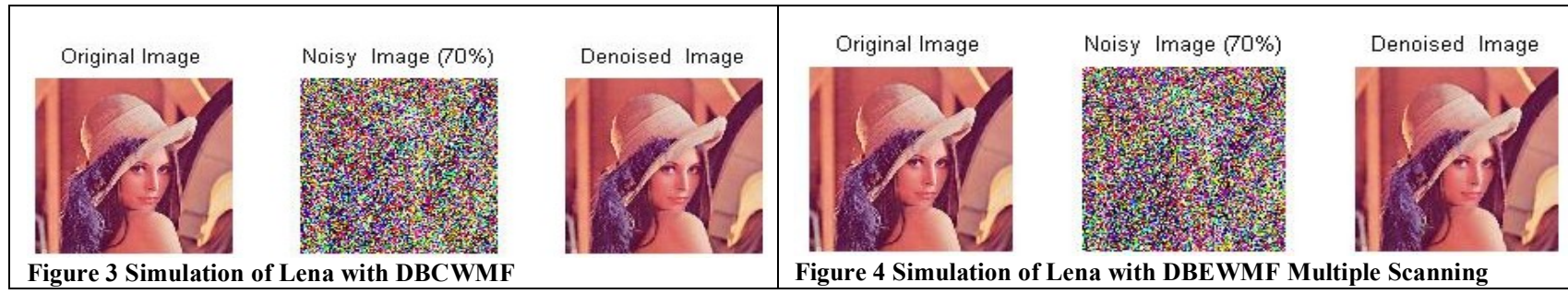

Table 1 Comparison of MSE

\begin{tabular}{|l|l|l|}
\hline \multirow{2}{*}{$\begin{array}{l}\text { Noise } \\
\text { Density(\%) }\end{array}$} & MSE \\
\cline { 2 - 3 } & DBCWMF & $\begin{array}{l}\text { DBEWMF With } \\
\text { multiple scanning }\end{array}$ \\
\hline 10 & 4.9630 & 0.4968 \\
\hline 20 & 10.773 & 1.0669 \\
\hline 30 & 17.209 & 1.7413 \\
\hline 40 & 24.488 & 2.4621 \\
\hline 50 & 32.939 & 3.2656 \\
\hline 60 & 43.216 & 4.1783 \\
\hline 70 & 54.527 & 5.3641 \\
\hline 80 & 68.994 & 7.2934 \\
\hline 90 & 92.956 & 25.5585 \\
\hline
\end{tabular}

Table 2 Comparison of BER

\begin{tabular}{|l|l|l|}
\hline \multirow{2}{*}{$\begin{array}{l}\text { Noise } \\
\text { Density(\%) }\end{array}$} & BER \\
\cline { 2 - 3 } & DBCWMF & $\begin{array}{l}\text { DBEWMF With } \\
\text { multiple scanning }\end{array}$ \\
\hline 10 & 0.0243 & 0.0195 \\
\hline 20 & 0.0264 & 0.0209 \\
\hline 30 & 0.0280 & 0.0219 \\
\hline 40 & 0.0292 & 0.0226 \\
\hline 50 & 0.0303 & 0.0233 \\
\hline 60 & 0.0315 & 0.0239 \\
\hline 70 & 0.0325 & 0.0245 \\
\hline 80 & 0.0336 & 0.0253 \\
\hline 90 & 0.0352 & 0.0294 \\
\hline
\end{tabular}


Image De-noising By Decision Based Expanded Window Median Filter Using Multiple Scanning

\begin{tabular}{|c|c|c|}
\hline \multirow{2}{*}{$\begin{array}{l}\text { Noise } \\
\text { Density }(\%)\end{array}$} & \multicolumn{2}{|l|}{ PSNR } \\
\hline & DBCWMF & $\begin{array}{l}\text { DBEWMF With } \\
\text { multiple scanning }\end{array}$ \\
\hline 10 & 41.1733 & 51.169 \\
\hline 20 & 37.8074 & 47.849 \\
\hline 30 & 38.783 & 45.722 \\
\hline 40 & 34.241 & 44.217 \\
\hline 50 & 32.953 & 42.991 \\
\hline 60 & 31.774 & 41.920 \\
\hline 70 & 30.764 & 40.835 \\
\hline 80 & 29.742 & 39.501 \\
\hline 90 & 28.448 & 34.055 \\
\hline
\end{tabular}

\begin{tabular}{|l|l|l|}
\multicolumn{4}{|c}{ Table 4 Comparison of SSIM } \\
\cline { 2 - 3 } $\begin{array}{l}\text { Noise } \\
\text { Density(\%) }\end{array}$ & SSIM & $\begin{array}{l}\text { DBEWMF With } \\
\text { multiple scanning }\end{array}$ \\
\hline 10 & 0.9762 & 0.9962 \\
\hline 20 & 0.9717 & 0.9915 \\
\hline 30 & 0.9658 & 0.9857 \\
\hline 40 & 0.9587 & 0.9784 \\
\hline 50 & 0.9497 & 0.9684 \\
\hline 60 & 0.9369 & 0.9551 \\
\hline 70 & 0.9196 & 0.9331 \\
\hline 80 & 0.8901 & 0.9096 \\
\hline 90 & 0.8067 & 0.8289 \\
\hline
\end{tabular}

Table 5 Comparison of IEF

\begin{tabular}{|l|l|l|}
\hline \multicolumn{2}{|l}{$\begin{array}{l}\text { Noise } \\
\text { Density(\%) }\end{array}$} & IEF \\
\cline { 2 - 3 } & DBCWMF & $\begin{array}{l}\text { DBEWMF } \\
\text { multiple scanning }\end{array}$ \\
\hline 10 & 461.071 & 905.488 \\
\hline 20 & 417.748 & 835.584 \\
\hline 30 & 361.993 & 761.987 \\
\hline 40 & 321.754 & 715.138 \\
\hline 50 & 272.425 & 666.695 \\
\hline 60 & 243.393 & 620.291 \\
\hline 70 & 194.639 & 560.395 \\
\hline 80 & 194.325 & 467.097 \\
\hline 90 & 107.351 & 193.841 \\
\hline
\end{tabular}

\begin{tabular}{|c|c|c|}
\hline \multirow{2}{*}{$\begin{array}{l}\text { Noise } \\
\text { Density }(\%)\end{array}$} & \multicolumn{2}{|l|}{ IQI } \\
\hline & DBCWMF & $\begin{array}{l}\text { DBEWMF With } \\
\text { multiple scanning }\end{array}$ \\
\hline 10 & 0.9316 & 0.9718 \\
\hline 20 & 0.8994 & 0.9396 \\
\hline 30 & 0.8625 & 0.9028 \\
\hline 40 & 0.8201 & 0.8605 \\
\hline 50 & 0.7711 & 0.8126 \\
\hline 60 & 0.7138 & 0.7561 \\
\hline 70 & 0.6478 & 0.6845 \\
\hline 80 & 0.5641 & 0.6678 \\
\hline 90 & 0.4303 & 0.5334 \\
\hline
\end{tabular}

Graphically it is clear that proposed algorithm DBEWMF is better than the existing algorithm[8]. As shown MSE and BER both are near to zero PSNR,SSIM,IQI,IEF are increasing and also contains improved values than standard filters.

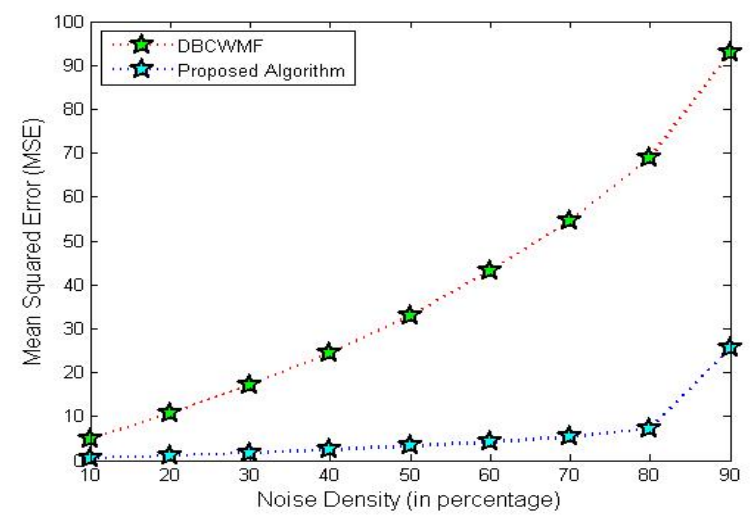

Figure 5 Comparison Graph of MSE

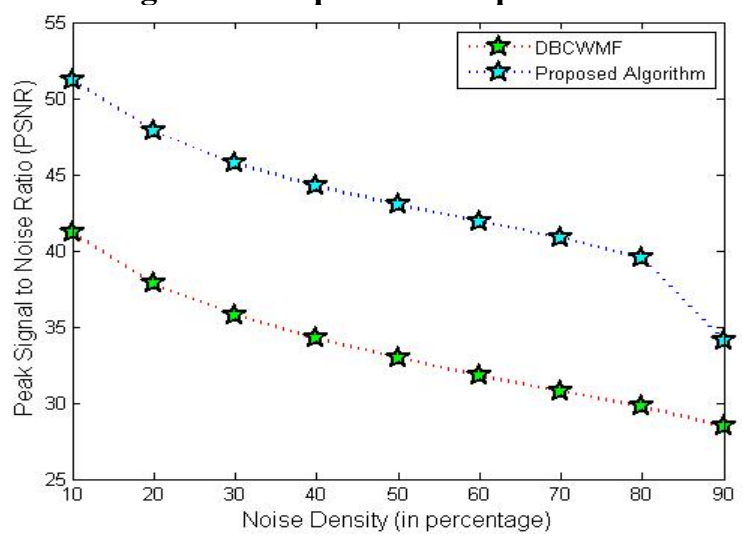

Figure 7 Comparison Graph of PSNR

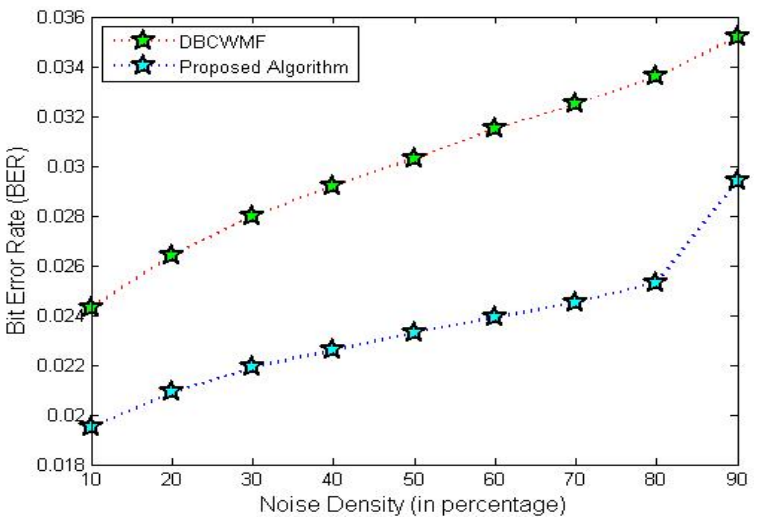

Figure 6 Comparison Graph of BER

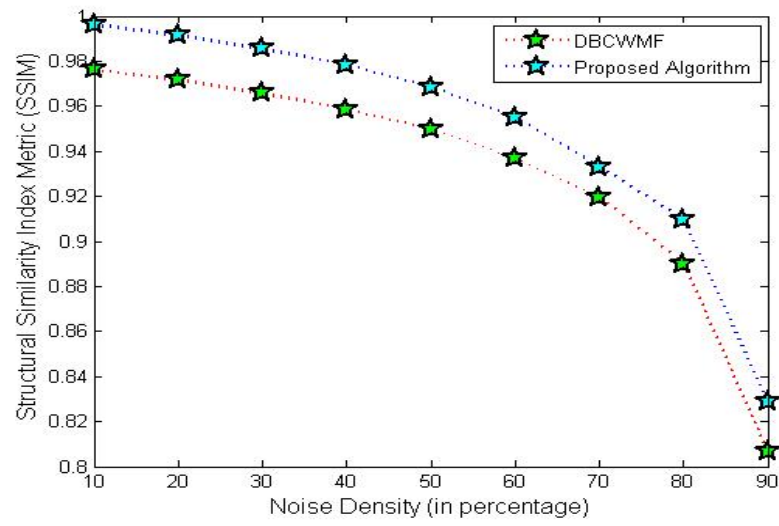

Figure 8 Comparison Graph of SSIM 


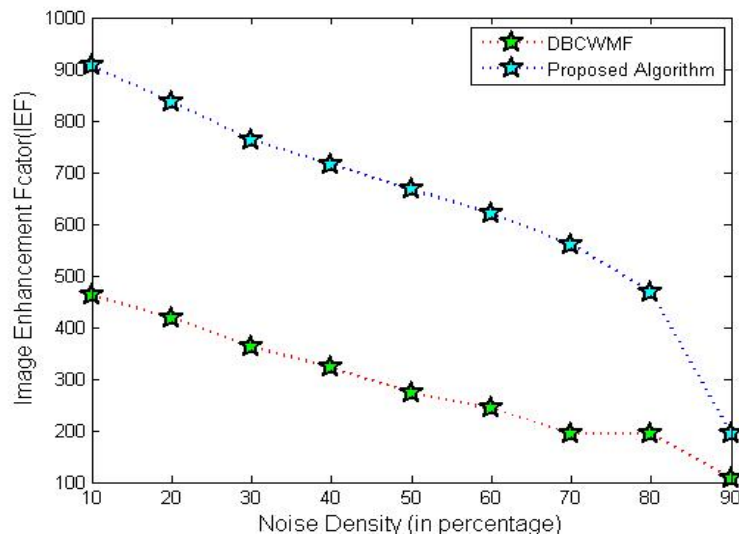

Figure 9 Comparison Graph of IEF

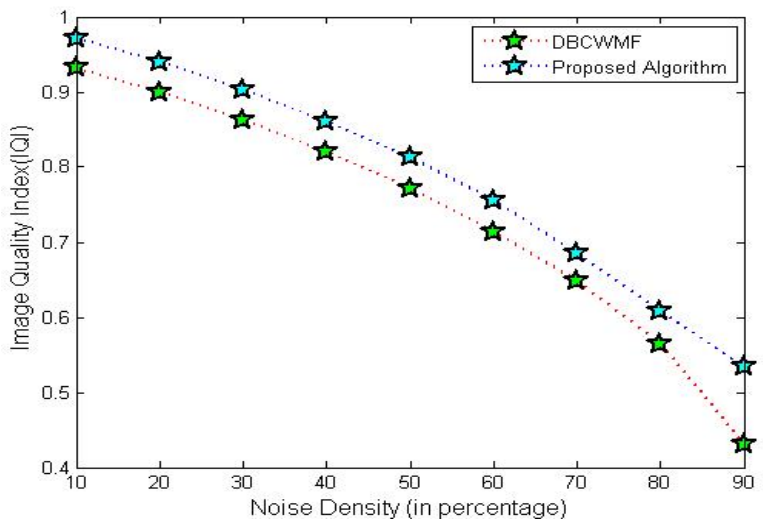

Figure 10 Comparison Graph of IQI

\section{Conclusion}

In this paper an efficient decision based expanded widow with median filter having multiple scanningto restore an image corrupted with high density salt and pepper noise is proposed. DBEWMF with multiple scanning algorithm consist of three stages. This algorithm operates only on noisy pixels and gives better result as low noise density as well as high noise density. It has been found that proposed algorithm comparatively provides better results in terms of MSE, BER, PSNR, SSIM,IQI,IEF.

\section{Acknowledgment}

This is to express my sincere gratitude to Mr. Vishal Kumar Arora, AssistantProfessor, Department of Computer Science andEngineering, SBS State Technical Campus, Ferozepur (Punjab), India, for sparking in me the enthusiasm and initiative to discover and learn. I am truly thankful to him for guiding me through the entire paper and being as a motivator in this learning curve.

\section{References}

[1] Lei Zhang a, _, WeishengDong a,b, DavidZhang a, GuangmingShi, "Two-stage image denoising by principal component analysis with local pixel grouping,"Elsevier 2009

[2] Charu Khare, Kapil Kumar Nagwanshi, "Implementation and Analysis of Image Restoration Techniques", International Journal of Computer Trends and Technology- May to June Issue 2011, ISSN:2231-2803

[3] Pitas, I.: Digital Image Processing Algorithms and Applications.Wiley, Hoboken (2000)

[4] Pomalaza-Racz, C.A., Macgillem, C.D.: An adaptive non linear edge preserving filter. IEEE Trans. ASSP 32(3), 571-576 (1984)

[5] Raymond H. Chan, Chung-Wa Ho, and Mila Nikolova, "Salt-and-Pepper Noise Removal by Median-Type Noise Detectors and Detail-Preserving Regularization, IEEE TRANSACTIONS ON IMAGE PROCESSING, VOL. 14, NO. 10, OCTOBER 2005

[6] Taeg Sang Cho et al. "Image Restoration by Matching GradientDistributions." IEEE Transactions on Pattern Analysis andMachine Intelligence 34.4 (2012): 683-694

[7] Nelly Pustelnik, Caroline Chaux, and Jean-Christophe Pesquet,"Parallel Proximal Algorithm for Image Restoration Using HybridRegularization - Extended Version, 2011,IEEE.

[8] Lei Zhang a, _Vivek Singh Bhadouria,DibyenduGhoshal, AbulHasanSiddiqi, "A new approach for high density saturated impulse noise removal using decision-based coupled window median filter," Accepted: 20 March 2013 Springer.

[9] S. Esakkirajan, T. Veerakumar, Adabala N. Subramanyam, and C. H. PremChand, "Removal of High Density Salt and Pepper NoiseThrough Modified Decision Based Unsymmetric" IEEE SIGNAL PROCESSING LETTERS, VOL. 18, NO. 5, MAY 2011

[10] GolamMoktaderDaiyan, M. A. Mottalib, Muhammad MizanurRahman, , , "High Performance Decision Based Median Filterfor Salt and Pepper Noise Removal in Images," 978-1-4673-4836-2/12, 2012 IEEE.

[11] T.Veerakumar1 S.Esakkirajan2 and Ila Vennila3: "Salt and Pepper Noise Removal in Video using Adaptive Decision Based Median Filter,"2011 International Conference on Multimedia, Signal Processing and Communication Technologies,2011 IEEE.

[12] Mr. Piyush a. Dhande1, dr. A. D. Gawande2, "comparative study on removal of high density salt and pepper noise through modified decision based unsymmetric trimmed median filter," international journal of pure and applied research in engineering andtechnology ijpret, 2014; volume 2 (9): 145-151

[13] DebanandaPadhiand SucharitaPadhi, “ Order Statistic Filters for removing Salt-and-Pepper Noise of Images: A Comparative Study ". international Journal of Advanced Research in Computer Science and Software Engineering,Volume 2, Issue 1, January 2012

[14] Idan Ram, Michael Elad, Fellow, IEEE, and Israel Cohen, Senior Member, IEEE, "Image Processing using Smooth Ordering of its Patches," IEEE 2012.

[15] K. Vasanth, VJawaharSenthilKumar, V Rajesh, Anitha, "Cascaded Decision Based Non Linear Filters for High Density Outlier Noise Removal in Images and Videos,"2013 International Conference on Circuits, Power and Computing Technologies [ICCPCT 2013], 978-1-4673-4922-2113, 2013 IEEE

[16] Pierrick Coup'e, Jos'e V. Manj'on,“Adaptive Multiresolution Non-Local Means Filterfor 3D MR Image Denoising,’IET iamge processing 2011 